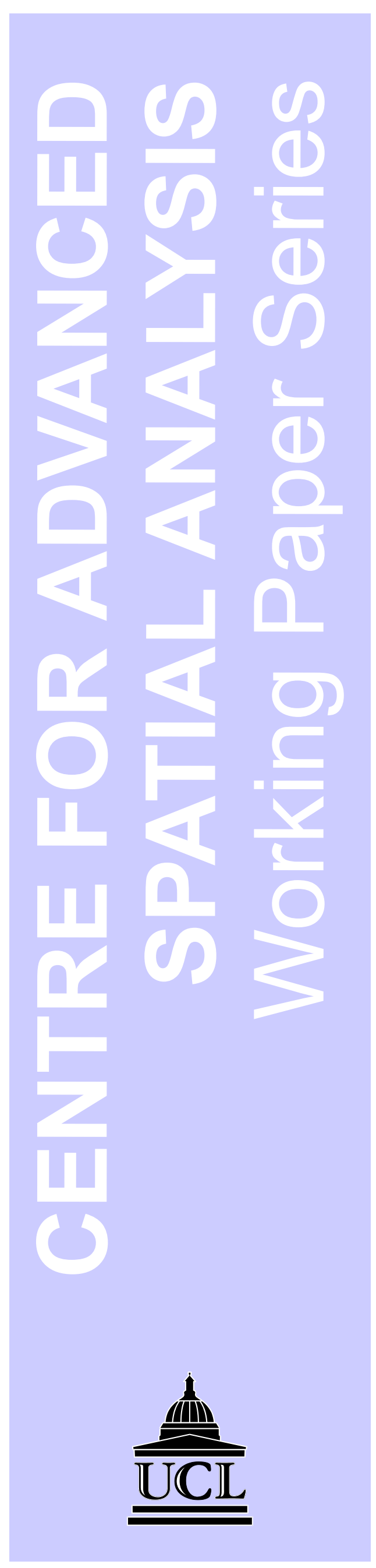

Cfisf

\title{
CELLULAR MODELS OF URBAN SYSTEMS
}

David O'Sullivan Paul M. Torrens 


\section{CASA}

Centre for Advanced Spatial Analysis

University College London

1-19 Torrington Place

Gower Street

London WC1E 6BT

Tel: +44 (0)207679 1812

Fax: +44 (0)20 78132843

Email: casa@ucl.ac.uk

http://www.casa.ucl.ac.uk

http://www.casa.ucl.ac.uk/cellularmodels.pdf

Date: June 2000

ISSN: 1467-1298

This paper appears in Bandini, S. \& Worsch, T. (eds) 2001, Theoretical and Practical Issues on Cellular Automata, Proceedings of the Fourth International Conference on Cellular Automata for Research and Industry (ACRI 2000), pages 108116, October 4-6, Karlsruhe, Germany, published by Springer-Verlag, London, ISBN 1-85233-388-X. This CASA Working Paper version is published and distributed under license from Springer-Verlag Ltd, London. 


\title{
Cellular Models of Urban Systems
}

\author{
David O’Sullivan Paul M. Torrens
}

June 2000

\begin{abstract}
Cellular automaton (CA) based models are increasingly used to investigate cities and urban systems. We discuss difficulties with this representation of human systems, and suggest that many modifications to simple CA introduced in modelling cities are responses to these problems. We propose a two-pronged approach to research. First, for operational model-building many variations on the $\mathrm{CA}$ theme are required and should be welcomed; and second theoretically motivated variations of the CA formalism are required so that the possible effects on model dynamic behaviour may be more systematically explored.
\end{abstract}

\section{Introduction}

Cellular automata (CA) have been applied to the simulation of an impressive range of urban phenomena. CA models have been used to study land use dynamics (Cecchini 1996, Webster \& Wu 1999a,b, White \& Engelen 1993, White, Engelen \& Uljee 1997); regional scale urbanization (Semboloni 1997, White \& Engelen 1997) and polycentricity (Wu 1998); urban socio-spatial segregation (Portugali 2000); development (Wu \& Webster 1998); location analysis (Benati 1997); urbanism (Sanders, Pumain, Mathian, Guérin-Pace \& Bura 1997); and urban growth and sprawl (Batty 1998, 1999, Clarke, Hoppen \& Gaydos 1997). This is a wide-ranging and sophisticated list, and the technique has a great deal to offer as a tool for investigating cities. However, the employment of $\mathrm{CA}$ in urban simulations often entails substantial departures from the original formal structure of CA described by von Neumann, Ulam, Conway, and Wolfram. Although the application of CA to urban systems seems natural and intuitive, this is not in itself sufficient justification for their use (Couclelis 1985), and in this paper we consider some of the modelling issues involved in constructing such models.

In section 2 we discuss how CA-type models can be held to represent reality. Section 3 then considers some of the many ad hoc modifications to the CA formal- 
ism which have been introduced by urban modellers in a bid for realism (see also White 1998). We suggest in section 4 that many of these operational variations may be seen as responses to the representational issues we have raised, and propose that a wider family of CA-like models is required.

\section{CA models and the real geographical world}

In this section we examine some of the more common modes of abstraction employed when CA are applied to geographical systems.

\subsection{The meaning of cells and the meaning of rules}

The CA formalism proposes a regular lattice of identical cells, each of which may be in one of a finite number of discrete states at discrete time steps in its evolution. System dynamics are determined by transition rules which map the current state of a cell's neighbourhood at time $t$, to an outcome cell state at time $[t+1]$. A key question in developing any urban CA model is then: "What do the cells represent? And, given what they represent, what is an adequate set of allowed cell states?" The answer to these questions affects the construction, meaning, and interpretation of transition rules.

In geographic applications, cells are usually treated very much as in the formal CA case, as cells in a two-dimensional grid-based lattice (although see Phipps 1989, where a hexagonal lattice is used). This approach is influenced by the digital, pixelised nature of data from remote-sensed and other sources, and also by the convenience of programming and implementing grid-based structures. Since urban systems can only be regarded as grid-based at the most general level of abstraction, this necessitates a similarly abstract approach to defining cell states. The idea of the cell state may become a more or less complex classification of land uses, land values, land covers, population densities, and so on. Various categories of residential, commercial, and industrial use are typical. At a more abstract level, cell states may be characterised in a binary fashion as developed or not developed. The grid representation, together with such an abstract cell classification, compromises the claim of the technique to be micro-simulation, except in the most general sense.

Such frameworks also beg the question of the meaning of transition rules. Land uses do not 'mutate' like cell cultures on a microscope slide. Rather, human agents - developers, firms, financiers, regulatory authorities, landlords, tenants, homebuyers - manœuvre, collaborate, and compete to change the city for their own 
purposes. The combination of their activities is what causes 'state transitions'. Seen in this light, it may be difficult to justify a pure state transition approach to rules in a cellular land use model. Rules must be understood as somehow embodying all this human activity. This raises the question of why human agents are not explicitly represented, and suggests that agent-based approaches may sometimes be more appropriate. There are cases in which cell transition rules may be appropriate, the most obvious being traffic simulation (Chopard, Luthi \& Queloz 1996, Nagel \& Schreckenburg 1992, Wahle, Neubert \& Schreckenburg 1999) where cells are 'vehicle-sized' segments of road, and cell states represent occupancy by vehicles. Significantly, this is a realm where human autonomy is strongly constrained by rules of the road and the spatial structure of the (now non-regular) lattice.

This brief outline indicates that there is a two-way relationship between what cells represent and how rules are defined, and what they represent. More objectbased approaches to cells - where cells represent land parcels, administrative areas, or even individual buildings - almost invariably require non-regular lattices, but may ease the problem of defining transition rules. Conversely, more typical, abstract representations which have been employed, may require a more subtle interpretation of the model transition rules.

\subsection{Local and global interactions}

By simulating the emergence of global structures from local elements, CA have much to offer urban simulation. Intuitively, we understand that many urban phenomena are structured 'from the bottom-up': air pollution, traffic congestion, neighbourhood upgrading and decline, and so on. The local-global relation in urban environments is emphatically of importance in models.

However, much of the emphasis in CA approaches (and in complexity science in general) has been on the emergence of global structure from local events. The opposite is also possible: global structure may be a constraint on local interaction. Many urban phenomena simply do not emerge solely from local interactions. Transport infrastructure and planning systems are obvious examples. This implies a need to consider non-local interactions in urban systems, which cuts across the formal logic of CA. Where CA models are decision support systems for policy makers this may actually be a happy coincidence, insofar as many policy interventions are non-local, and these can be inserted into models as external constraints or non-uniformities in the applicability of transition rules. Generally, however, the issue cannot be ducked in this way. Indeed, development of appropriate model 
structures in the urban case may offer significant insights for the use of CA in other domains, where similar observations apply.

\subsection{Time in CA models: synchronous and asynchronous update}

The representation of time in CA models is also of interest. Discretised 'time-steps' are at odds with the fluidity of temporal activity in reality, and the synchronous update of cell states is clearly questionable. Experiments with asynchronous update of cell states in abstract CA suggest that the dynamic implications of departure from synchronous update may be significant (Bersini \& Detours 1994). This suggestion is supported by findings on path dependency and lock-in, which have received much attention in economics (Arthur 1989), and were also central to the early transfer of ideas from complexity science to regional modelling (Allen \& Sanglier 1979), but may be missed in any straight-forward application of CA to urban simulation.

There are also difficult questions to be answered about the representation of events at many different temporal scales. The treatment of time in rigid CA raises problems for their application to the simulation of urban systems. In particular, questions about the spatio-temporal scale of models are raised, which are difficult to answer without circular reference back to the transition rules - since the length of a time-step determines how much change may occur in a single transition, which depends on the meaning of cell states and transition rules. A further practical difficulty is that an overlap of multiple processes operating at many different timescales (from the ecological, even geological, to the diurnal) is evident in cities.

\section{Adaptations of the $\mathrm{CA}$ formalism to the modelling of cities}

We now discuss efforts to fit CA to real urban systems and the resultant changes to the rigid CA formalism. These are introduced in order of the increasing degree to which they seem to depart from that formalism.

\subsection{Discrete, continuous and multivariate state variables}

Although introducing ten discrete cell states does not radically alter matters (von Neumann's replicator had 29 cell states), it is unclear how far we can take this. In general, differences between cell states are held to be qualitative (residential use 
rather than industrial, for example). However, it can be difficult to reduce all of the activity in an urban cell to a single discrete description. This is particularly true of relatively coarse grids (at say $100 \mathrm{~m}$ or more resolution). There is bound to be 'mixing' across cells, implying a fuzzy description of cell states. It is also difficult to represent some very significant features of the urban environment - transport networks and rivers being the foremost examples - in a grid-based cellular world.

Multivariate cell states may be more problematic, especially if rules are constructed so that each variable is updated separately according to inter-related but distinct processes. If the resulting transition table remains unambiguous, it may be argued that all that is really lost is a user's ready understanding of how changes to rules affect outcomes. In operational cases this may be less important than an ability to easily interpret model outcomes. Where CA are intended as a vehicle for interrogating urban theory this may be a significant advantage, since theories about various facets of urban life can be combined, and the implications of their interaction examined. This may then be an area where the modifications of urban modellers using CA are justified in both pragmatic and theoretical terms, without much damage being done to the formalism.

\subsection{Neighbourhood sizes and 'action-at-a-distance'}

In a formal CA, every state change must be local, and there is no allowance for action-at-a-distance. In a strict $\mathrm{CA}$ it is implied that the dynamics take care of distance effects simply because growth and decline appear as spatial diffusion. However, for this to be valid, the spatio-temporal scale of the model must be correct: where a cell transition can represent the conversion of a tract of land of (say) 10000 square metres to a new use in a single time step (so that by implication a time step is a period of the order of a year), it is clearly unrealistic to admit only local interactions. Researchers have tinkered with the formal treatment of CA neighbourhoods in a bid to include the flexibility of action-at-a-distance. Often a very large neighbourhood is used in lieu of immediate 4 or 8 cell neighbourhoods. For example, White \& Engelen (1993) introduce a 113 cell neighbourhood in their model of land use dynamics. Although this represents a dramatic modification of the lattice, it does not depart from the strict formalism provided cell neighbourhoods are spatially stationary, that is, similar at each location. 


\subsection{Non-regular lattices}

In practice, few urban CA models retain a spatially stationary lattice. The issue of regularity is often addressed conservatively by the introduction of 'fixed' cell states. Bodies of water and undevelopable land are obvious examples which effectively introduce irregularity and asymmetry (Clarke et al. 1997, White \& Engelen 1997). Fixed cells may also be introduced to 'protect' the model from edge effects (White et al. 1997). Traffic models deliberately employ non-regular lattices because the traffic system's spatial structure is non-regular (Chopard et al. 1996, Nagel \& Schreckenburg 1992, Wahle et al. 1999). The kind of object-based cell suggested in section 2.1 naturally leads to asymmetric and non-regular lattice structures, and the resulting models lie somewhere between CA and boolean networks (Kauffman 1984), albeit with spatially stationary rules.

\subsection{Asynchronous transition rules}

Truly asynchronous cell update in urban CA is unusual. However, some models incorporate forms of asynchronous determination of cell state changes into a synchronously updating framework. In the work of Portugali (2000) on intra-urban migration, queues of agents who wish to enter or leave the city are sequentially allocated to available 'properties'. Similarly in models of land use dynamics (White \& Engelen 1993, 1997, White et al. 1997), a CA sequentially determines the spatial allocation of land use transitions, determined outside the model. In both these cases, the departure from synchronous operation is partly a response to the fact that the region is embedded in a wider world, and is not a closed system. In another example, Wu (1999) introduces asynchronous operation as a fundamental aspect of the system dynamics, when he relates a model of urban development driven by the appearance of 'investment niches', to the notion of self-organised criticality. Whatever the motivation for introducing asynchronous cell update, the fundamental dynamics of such systems are not at all clear, and may require investigation.

\section{Does any of this matter?}

It should be made clear that the foregoing is not intended as criticism of the developers of cellular urban models. Many modifications to the CA formalism cited in section 3 are reasonable responses to the representational difficulties discussed in section 2. This is set out in figure 1. It is evident that there is no simple map- 


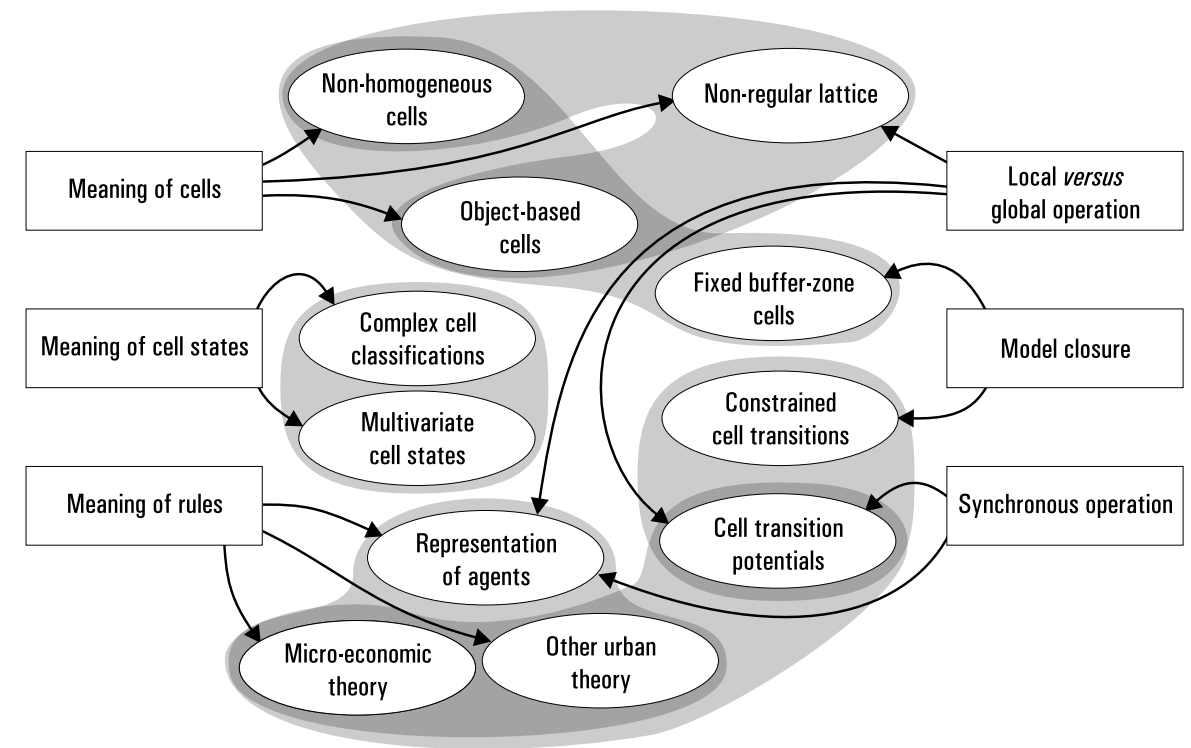

Figure 1: Various urban CA-based model variations (ovals) as responses to the representational problems of strict CA (rectangles). Tentative groupings of the variations are also shown

ping between problems and responses, nor would we expect one, since the purpose of most of the model-building efforts recorded here is to achieve some measure of representational realism, and different researchers have found various combinations of approaches appropriate. Our brief overview is not exhaustive, but does demonstrate the rapidity with which innovative new model structures are being developed, and also that some common combinations of $\mathrm{CA}$ variant are emerging.

Perhaps the most pertinent question is 'so what?!'. Does it matter that practitioners are moving away from the elegant, formal simplicity of conventional CA? The answer must be 'yes... and no.' The negative response is perfectly legitimate from the perspective of developing workable, convincing simulations of cities and urban systems which are useable in the real world. The important criteria for such operational models must be the extent to which model behaviour is theoretically plausible and therefore believable by practitioners and potential users, since this will strongly affect the prospects for their adoption and use.

However, there is another perspective where the answer to our question must be that yes, it does matter. Early in the discussion of CA-based urban models by the academic community, Helen Couclelis (1985, page 588) commented that 
"all the simplifying assumptions of the basic cell-space model could be relaxed in principle: in practice of course, the result would be forbiddingly complex." She was suggesting that one of the attractions of $\mathrm{CA}$ is the potential they provide for insights into the relationships between processes at local scales and structures at global scales. Such insight, apart from its pedagogic value, also raises the possibility of a deeper understanding of the fundamental dynamics of spatial systems. But, as Couclelis's remark indicates, any insights which might be obtained are rapidly clouded by the ever more complicated refinement of additional model elements.

There is no obvious simple way around this dilemma, but we wish to tentatively suggest an approach which we hope to develop in more detail over time. The reason that the CA formalism has exerted such fascination in so many fields, is its high level of generality. When it comes to applying the formalism, however, "it is necessary to use more complex CA" (White 1998, page 112), with a resulting loss in generality of the insights, but a gain in the direct applicability of the models to real systems. One response might be for those interested in exploring the dynamics of spatial systems in more general ways, to develop some well-defined, specific departures from the CA formalism. A preliminary list of possibilities might include:

- Strict formal CA with a small family of geographical process rules Theoretical exploration of the behaviour of urban CA would be much assisted by agreement on a limited set of typical process rules whose behaviour could then be thoroughly explored and characterised. Segregation, growth, aggregation and diffusion processes are obvious candidates.

- Cellular models with irregular lattice structures This concept is foreshadowed by Takeyama \& Couclelis's (1997) Geo-Algebra, and brought into clearer focus by the graph-based CA (O’Sullivan forthcoming). Such models might also be capable of modifying their lattice structure as a response to neighbourhood states (Semboloni forthcoming, has presented an example).

- Agents in cellular models The rules of a CA in an urban system ultimately reflect the behaviour of various human agents, and in many cases modelling the agents themselves seems more plausible. Portugali's (2000) Free Agents in Cellular Space model is a working example of this approach.

- Asynchronous cell update The limitation of CA models to synchronous update is problematic, and research into alternatives is required. One possibility seems likely to be using Petri nets in the definition of cell transition rules 
(see Gronewold \& Sonnenschein 1998).

The purpose of focusing on particular variants and extensions of the CA formalism is to enable research into the general spatial dynamics of such systems, so that some of the potential for insight promised by initial encounters with CA can be regained. An example of such research into graph-based CA is provided by O'Sullivan (forthcoming, 2001).

\subsection{Conclusions}

The approach we have advocated is at a preliminary stage. However, we feel that there is an urgent need for explicit development of a two-pronged approach to the study of dynamic complexity in cities, urban systems and spatial systems more generally. This approach acknowledges the need for development of whatever model structures appear to be required for the realistic representation of cities in operational models, but also advocates that these developments be formalised into a family of models appropriate for exploring the spatial dynamics of such models in a more general sense. Operational models might then come to be seen as the application of well understood formalisms, which reveal how the mechanisms in the various formalisms act in concert, rather than as a rapidly proliferating and bewildering array of only loosely related examples.

\section{References}

Allen, P. M. \& Sanglier, M. (1979), 'A dynamic model of growth in a central place system', Geographical Analysis 11(3), 256-272.

Arthur, W. B. (1989), 'Competing technologies, increasing returns, and lock-in by historical events', Economic Journal 99(394), 116-131.

Batty, M. (1998), 'Urban evolution on the desktop: simulation with the use of extended cellular automata', Environment and Planning A 30, 1943-1967.

Batty, M. (1999), 'Modelling urban dynamics through GIS-based cellular automata', Computers Environment and Urban Systems 23, 205-233.

Benati, S. (1997), 'A cellular automaton for the simulation of competitive location', Environment and Planning B 24, 175-192. 
Bersini, H. \& Detours, V. (1994), Asynchrony introduces stability in cellular automata based models, in R. A. Brooks \& P. Maes, eds, 'Artificial Life IV: Proceedings of the Fourth International Workshop on the Synthesis and Simulation of Living Systems', The MIT Press, Cambridge, MA \& London, pp. 382-387.

Cecchini, A. (1996), 'Urban modelling by means of cellular automata: generalised automata with the help on-line (AUGH) model', Environment and Planning B: Planning \& Design 23(6), 721-732.

Chopard, B., Luthi, P. O. \& Queloz, P.-A. (1996), 'Cellular automata model of car traffic in a two-dimensional street network', Journal of Physics A 29, 23252336 .

Clarke, K. C., Hoppen, S. \& Gaydos, L. (1997), 'A self-modifying cellular automaton model of historical urbanization in the San Francisco Bay area', Environment and Planning B: Planning \& Design 24, 247-261.

Couclelis, H. (1985), 'Cellular worlds: a framework for modeling micro-macro dynamics', Environment and Planning A 17, 585-596.

Gronewold, A. \& Sonnenschein, M. (1998), 'Event-based modelling of ecological systems with asynchronous cellular automata', Ecological Modelling 108, 3752.

Kauffman, S. A. (1984), 'Emergent properties in random complex automata', Physica D 10, 145-156.

Nagel, K. \& Schreckenburg, M. (1992), 'A cellular automaton model for freeway traffic', Journal de Physique 2, 2221ff.

O'Sullivan, D. (forthcoming), 'Graph-cellular automata: a generalised, discrete urban and regional model', Environment and Planning B: Planning \& Design.

O'Sullivan, D. (forthcoming, 2001), 'Exploring spatial process dynamics using irregular cellular automaton models', Geographical Analysis.

Phipps, M. (1989), 'Dynamical behavior of cellular automata under the constraint of neighborhood coherence', Geographical Analysis 21(3), 197-215.

Portugali, J. (2000), Self-Organization and the City, Springer-Verlag, Berlin.

Sanders, L., Pumain, D., Mathian, H., Guérin-Pace, F. \& Bura, S. (1997), 'SIMPOP: A multiagent system for the study of urbanism', Environment and Planning B: Planning \& Design 24, 287-305.

Semboloni, F. (1997), 'An urban and regional model based on cellular automata', Environment and Planning B 24, 589-612. 
Semboloni, F. (forthcoming), 'The growth of an urban cluster into a dynamic selfmodifying spatial pattern', Environment and Planning B: Planning \& Design.

Takeyama, M. \& Couclelis, H. (1997), 'Map dynamics: integrating cellular automata and GIS through Geo-Algebra', International Journal of Geographical Information Science 11, 73-91.

Wahle, J., Neubert, L. \& Schreckenburg, M. (1999), A dynamic route guidance system based on real traffic data, in 'Sixth International Conference on Computers in Urban Planning and Urban Management', Venice, Italy. Online at http://www.iuav.unive.it/stratema/cupum/.

Webster, C. J. \& Wu, F. (1999a), 'Regulation, land use mix and urban performance part 1. Performance', Environment and Planning A 31(8), 1433-1442.

Webster, C. J. \& Wu, F. (1999b), 'Regulation, land use mix and urban performance part 2. Theory', Environment and Planning A 31(9), 1529-1547.

White, R. (1998), 'Cities and cellular automata', Discrete Dynamics in Nature and Society 2, 111-125.

White, R. \& Engelen, G. (1993), 'Cellular automata and fractal urban form', Environment and Planning A 25, 1175-1199.

White, R. \& Engelen, G. (1997), 'Cellular automata as the basis of integrated dynamic regional modelling', Environment and Planning B: Planning \& Design 24, 235-246.

White, R., Engelen, G. \& Uljee, I. (1997), 'The use of constrained cellular automata for high-resolution modelling of urban land use dynamics', Environment and Planning B: Planning \& Design 24, 323-343.

Wu, F. (1998), 'An experiment on the generic polycentricity of urban growth in a cellular automatic city', Environment and Planning B: Planning \& Design 25, 731-752.

Wu, F. (1999), A simulation approach to urban changes: experiments and observations on fluctuations in cellular automata, in P. Rizzi, ed., 'Sixth International Conference on Computers in Urban Planning and Urban Management', Venice, Italy. Online at http://www.iuav.unive.it/stratema/cupum/.

Wu, F. \& Webster, C. J. (1998), 'Simulation of land development through the integration of cellular automata and multicriteria evaluation', Environment and Planning B: Planning \& Design 25, 103-126. 